
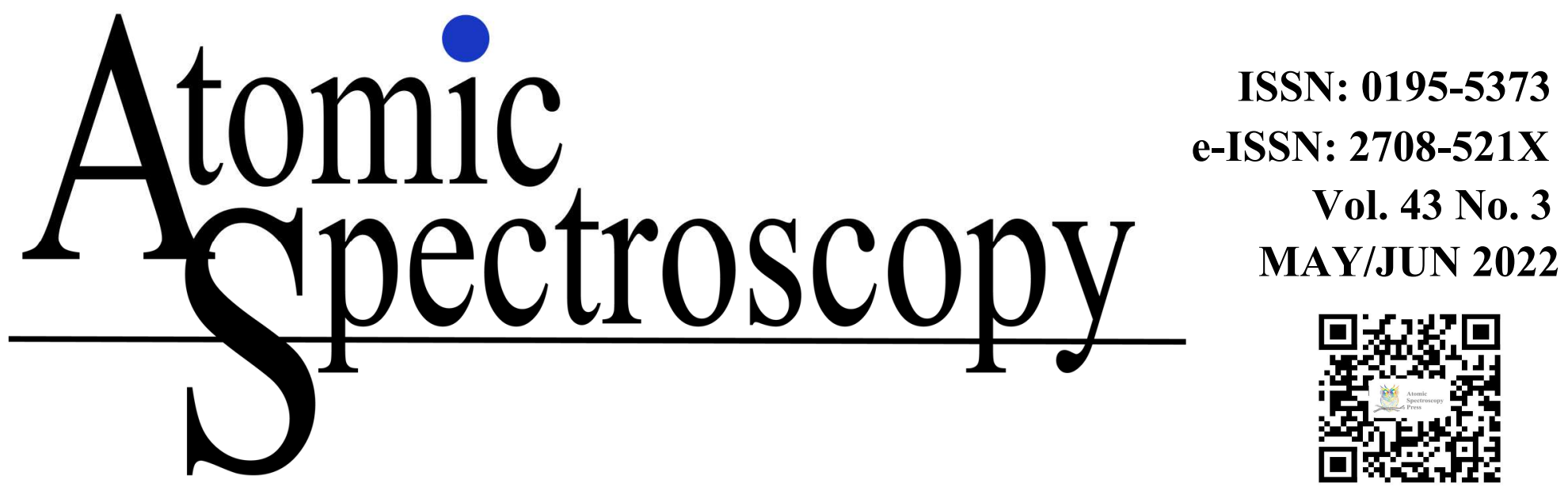

www.at-spectrosc.com

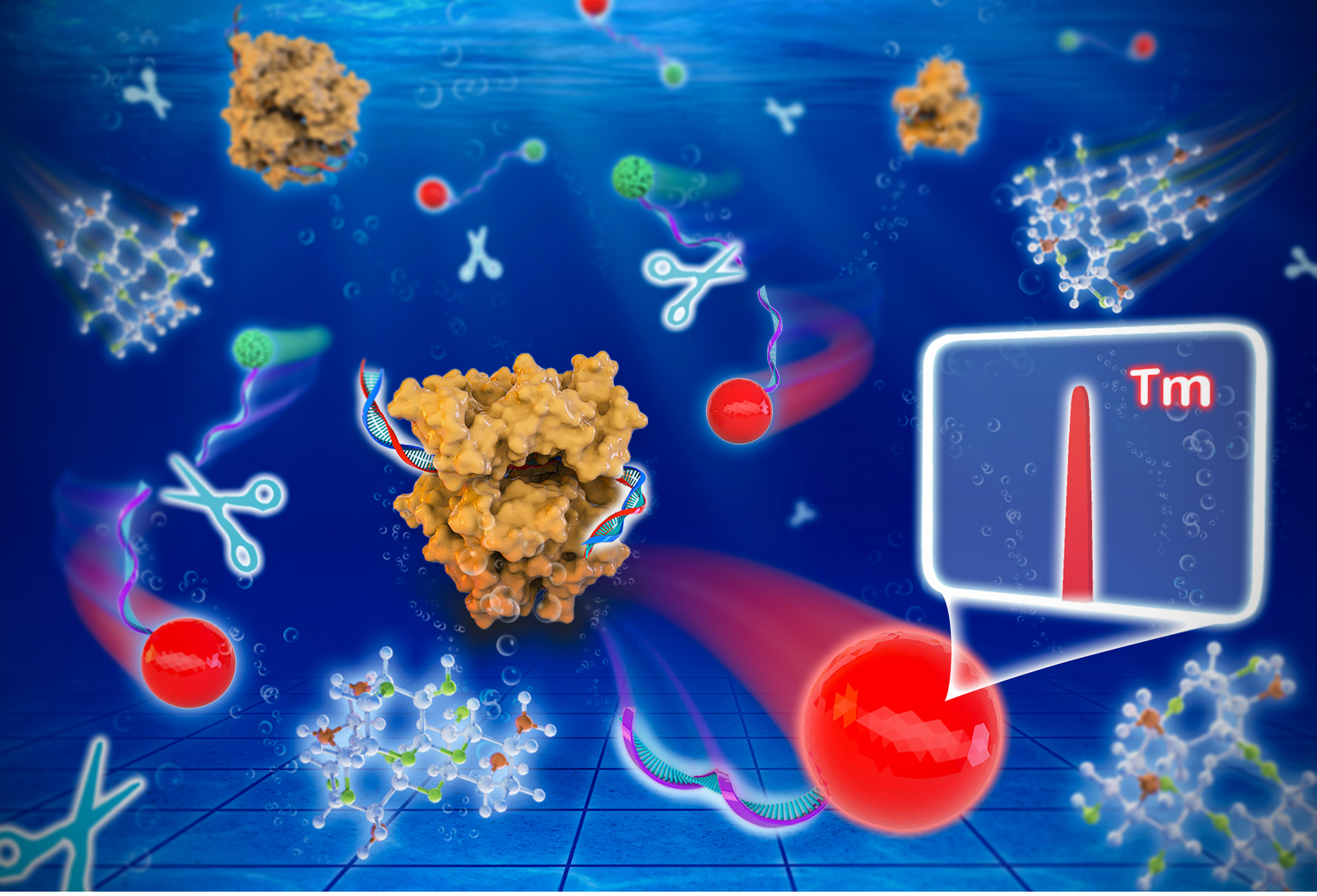

\section{Cover Feature:}

Elemental Probe-Based CRISPR/Cas12a Biosensing for Sensitive Tobramycin Detection

Ziqiang Deng, Jianyu Hu, Rui Liu, and Yi Lv

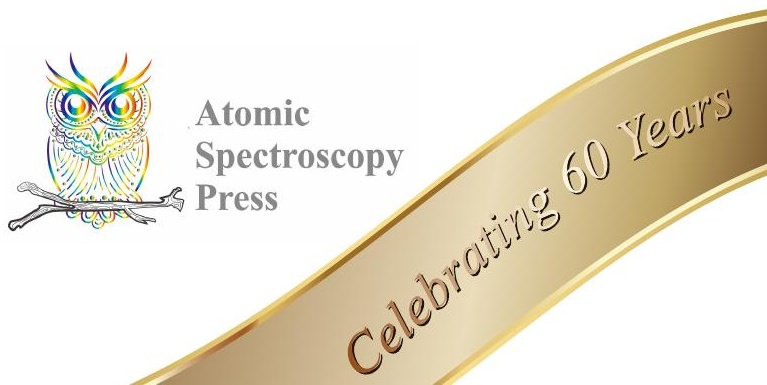




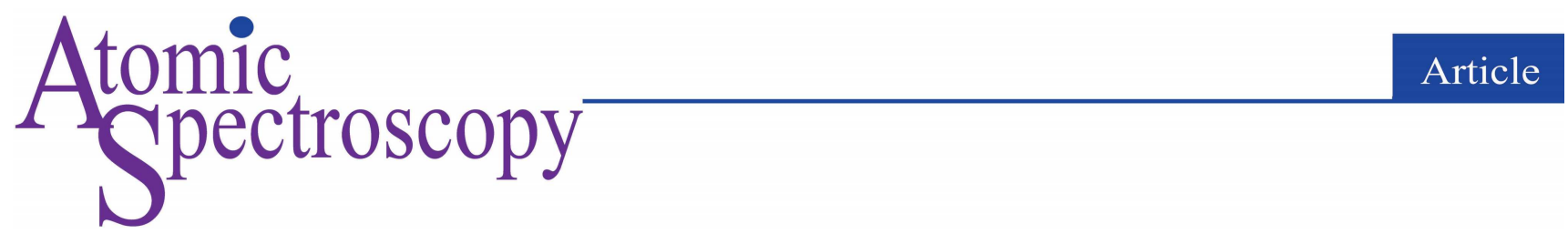

\title{
Elemental Probe-Based CRISPR/Cas12a Biosensing for Sensitive Tobramycin Detection
}

\author{
Ziqiang Deng, ${ }^{\text {a Jianyu Hu, }}{ }^{\mathrm{b}}$ Rui Liu, ${ }^{\mathrm{a}, *}$ and $\mathrm{Yi} \mathrm{Lv} v^{\mathrm{a}, \mathrm{b}}$ \\ ${ }^{a}$ Key Laboratory of Green Chemistry \& Technology, Ministry of Education, College of Chemistry, Sichuan University, Chengdu 610064, P. R. China \\ ${ }^{\mathrm{b}}$ Analytical \& Testing Center, Sichuan University, Chengdu 610064, P. R. China
}

Received: October 23, 2021; Revised: December 02, 2021; Accepted: December 02, 2021; Available online: December 27, 2021.

DOI: 10.46770/AS.2021.1013

\begin{abstract}
researchers exploring the potential of this system for detecting various other targets. Herein, we proposed a novel CRISPR/Cas biosensing based Tobramycin (TOB) detection method. Combining with the aptamer recognition and element labeling strategy, highly sensitive and specific detection of TOB is realized. Due to the powerful elemental analysis capabilities of inductively coupled plasma mass spectrometry (ICP-MS) and the excellent signal amplification effect of CRISPR/Cas, the linear concentration range for TOB was 2.8-100 nM in this method, whereas the detection limit was $0.83 \mathrm{nM}$. The proposed method has been successfully applied in the analysis of actual water samples.
\end{abstract}

ABSTRACT: The precise targeting and excellent specific cleave capability of CRISPR/Cas (clustered regularly inters-paced short palindromic repeats-associated proteins) have gained much research interest. In addition to nucleic acid detection,

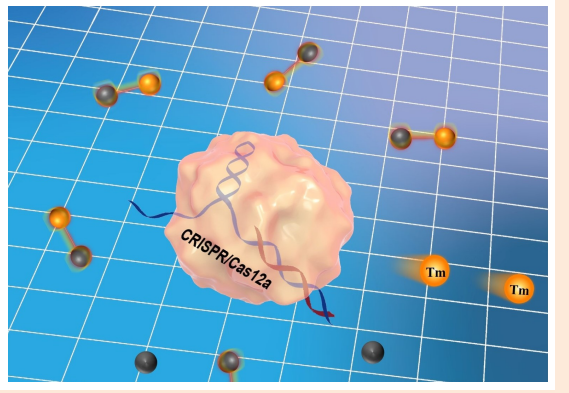

\section{INTRODUCTION}

Antibiotics have brought huge benefits to human society in the past nearly 100 years. ${ }^{1}$ However, with the extensive use of antibiotics in aquaculture and other fields, overused antibiotics are discharged into nature and become environmental contaminants. $^{2}$ Tobramycin (TOB), an aminoglycoside antibiotic, is mostly used for infections caused by gram-positive organisms along with some gram-negative. ${ }^{3}$ The structure of TOB is shown in Table 1, the amino groups have a basic protonation range from 5.67 to 9.29 , so there are five protonation equilibria (pKa) in basic or acidic conditions ${ }^{4}$, which make it has a good water solubility and broad antibacterial spectrum.

TOB is widely used in veterinary medicine for its low price and remarkable antibacterial effect. ${ }^{5}$ The European Commission has established strict maximum residue limits(MRL) for aminoglycoside antibiotics in several animal-derived foods, and set the maximum residue limit of tobramycin in milk as 200 $\mu \mathrm{g} \cdot \mathrm{kg}^{-1} .{ }^{6}$ Due to the water solubility and wide application range of TOB, TOB is easy to accumulate in natural water bodies. Once TOB accumulates in the human body as a result of migrating through the food chain, it must be metabolized by the liver and excreted through the kidneys, which causes a potentially irreversible toxic effect on the liver, as well as nephrotoxicity. ${ }^{7}$ Therefore, sensitive and accurate TOB testing is vital in clinical, environmental analysis and food safety monitoring.

Traditional antibiotic detection methods include microbial assay ${ }^{8}$ and immunoassay. ${ }^{9}$ Despite the facile operation and low-cost, the analytical performance of these two methods can't satisfy the demand of antibiotic detection, especially, the specificity and sensitivity need to be improved. In addition, it is difficult to meet the requirements of low concentrations detection. ${ }^{10}$ At present, chromatographic based technology is the mainstream of antibiotic detection, especially high-performance liquid chromatography (HPLC). ${ }^{11-13}$ Although the sensitivity and accuracy of separation technology based are excellent, the disadvantages such as complicated operations and pretreatment procedures are unavoidable.

In recent years, biosensors ${ }^{14-16}$ have developed rapidly in antibiotic detection, ${ }^{17}$ with advantages such as quick response, 
Table 1. Physico-chemical properties of tobramycin ${ }^{4}$

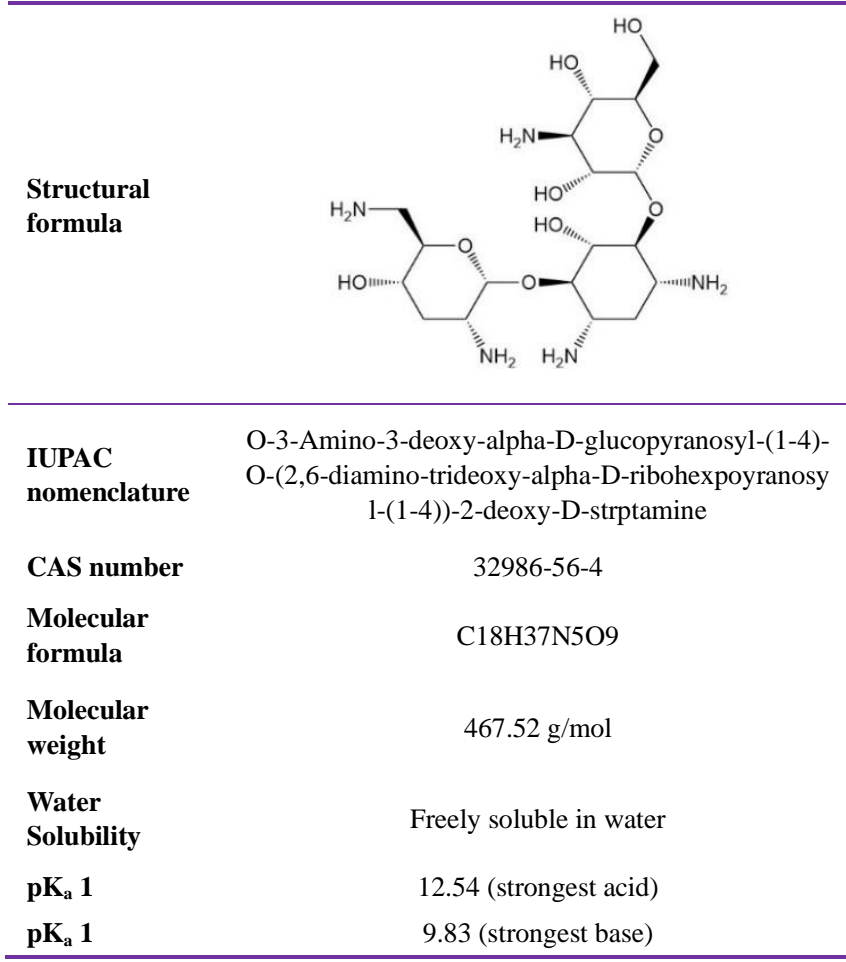

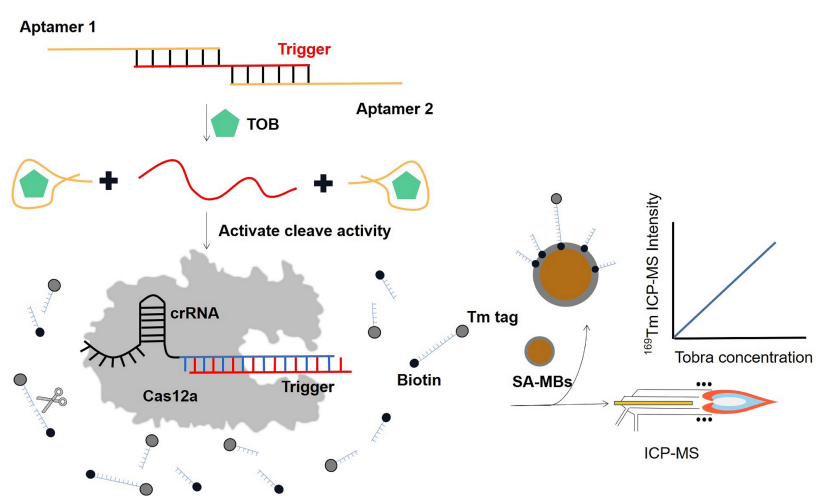

Fig. 1 Schematic diagram of the elemental probe-based CRISPR/Cas12a system for the detection of TOB.

high specificity, and excellent sensitivity. ${ }^{18}$ On the basis, the CRISPR/Cas system that won the 2020 Nobel Prize deserves attention in the field of antibiotic detection. Precise targeting and extremely high specific cleavage ability, these characteristics make the CRISPR/Cas system has potential to develop into a fast, simple, low-cost, and versatile biosensor. ${ }^{19-21}$ In the field of biomedical technology, CRISPR/Cas gene editing technology is tantamount to another major innovation after PCR(Polymerase chain reaction) technology. ${ }^{22}$ Although the application of the CRISPR/Cas system is mainly embodied in the field of biomedicine, the potential of the CRISPR/Cas system to radiate to other fields with nucleic acid as a medium cannot be ignored..$^{23}$

Taking into account the ease of programming ${ }^{24}$ and specific binding ability of DNA aptamer, DNA aptamer was used as mediation to discover the potential of the CRISPR/Cas system in antibiotic biosensing detection. In this work, among a series of Cas enzymes which widely used including type II (Cas9), type V (Cas12 and Cas14) and type VI (Cas13), ${ }^{25}$ we chose Cas12a, which can recognize and cleave single-stranded DNA (ssDNA) indiscriminately and rapidly. ${ }^{26}$ For the advantages of high sensitivity, wide dynamic range, especially multi-component quantification ability, ${ }^{27}$ inductively coupled plasma mass spectrometry (ICP-MS) has fantastic potential in metal element detection. ${ }^{28-31}$ With applying the strategy of element labeling, ${ }^{32-33}$ the sensitivity of ICP-MS ${ }^{34}$ is well integrated into our biosensor. ${ }^{35}$ Our work confirms that this biosensing method can meet the requirements for trace concentration TOB detection, and has the potential for the detection of a variety of antibiotics and other targets with corresponding aptamers.

\section{METHODS AND MATERIAL}

As shown in Fig. 1, in the presence of TOB, due to the stronger specific binding ability of the aptamer with TOB, the Trigger chain will be released from the locking structure, bind to Cas12a/crRNA, and activate the branch cleavage activity of Cas12a, the Tm probe was severely cleaved at this time, achieved the purpose of signal amplification. In the absence of TOB, Tm probe will be intact. By modifying the biotin on one side of the Tm probe, after the separation by SA-MBs and the magnetic stand, the biotin segment of the cleaved probe and the intact probe are removed, the Tm element-labeled part of the cleaved probe will be retained in the supernatant. The supernatant will be processed and sent to the ICPMS for quantitative detection of stable Tm isotopes. The detected ${ }^{169} \mathrm{Tm}$ signal strength is directly related to the concentration of Cas12a that activates the cleavage ability, and depends on how many priming sequences are released by the combination of tobramycin and the aptamer. Therefore, the final ${ }^{169} \mathrm{Tm}$ signal intensity correlates with the target TOB concentration.

Trigger Locking Structure and Tm Probe. In the detection system we designed, we used the locking structure of dual aptamer-Trigger, ${ }^{36}$ and used the ratio of Aptamer $^{10}$ 1: Aptamer 2: Trigger=2: 2: 1 in the hybrid preparation to ensure that the Trigger sequence could be completely locked by TOB aptamer ${ }^{23}$. The structure of the Tm probe is shown in Fig. 2, we replaced the commonly used "fluorescence-quenching group" 37 reporter probe with an element-labeled probe which had made gratifying progress in the study of metal ions and macrocyclic compounds $\mathrm{N}, \mathrm{N}^{\prime}, \mathrm{N}^{\prime}, \mathrm{N}^{\prime \prime \prime}$-tetraacetic acid chelate (DOTA) labeled biomolecules. ${ }^{38-39}$ Withing this method, combined with the signal amplification function of the indiscriminate branch cleaving characteristic of Cas12a, we successfully completed the effective detection of TOB in actual water sample. 


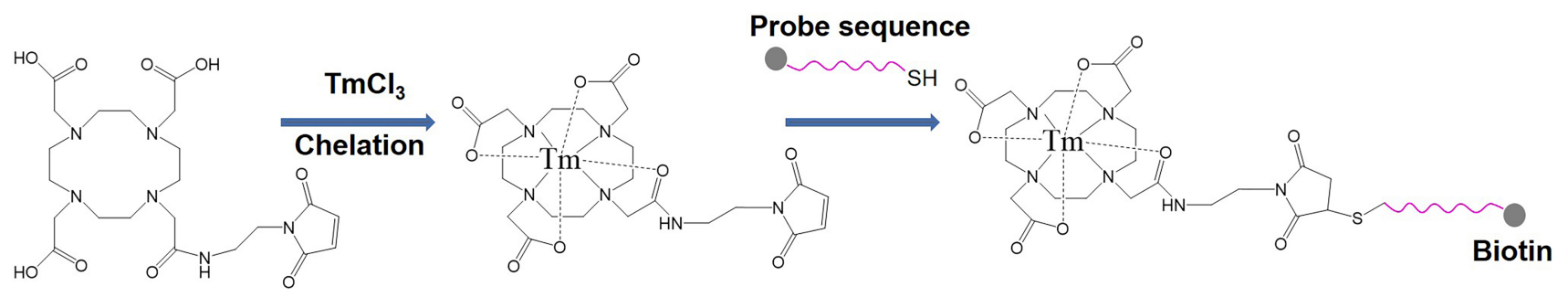

Fig. 2 Schematic diagram of the structure and preparation process of the Tm element probe.

Reagents, Nucleic Acid Sequence and Instruments. The materials and instruments employed included maleimide-modified N,N',N",N"'-tetraacetic acid chelate (MMA-DOTA) (Macrocyclics, Dallas, TX, USA), TmCl3.6H2O and $\mathrm{CH} 3 \mathrm{COONH} 4$ (Aladdin Biochemical Technology Co., Ltd., Shanghai, China), DEPC-treated denuclease water, SA-MBs, 3k Amicon Ultra-0.5 NMWL ultrafiltration tubes (Sangon Biotechnology Co., Ltd., Shanghai, China), LbCas12a (cpf1) with its corresponding 10×TOLO buffer 3 (Tolo Biotechnology Co. Ltd. Shanghai, China), and all antibiotics used in the experiment (Macklin Biotechnology Co., Ltd., Shanghai, China).

In addition, HEPES buffer (pH 7.4, 40 mM HEPES, $100 \mathrm{mM}$ $\mathrm{NaCl}, 20 \mathrm{mM} \mathrm{MgCl}_{2}$ ) used in CRISPR/Cas12a cleavage reactions use DEPC-treated denuclease water. Deionized water (DIW, $18.2 \mathrm{M} \Omega \cdot \mathrm{cm}-1$ ) is purified by Milli-Q ultrapure water system, and 2X B\&W buffer (10 mM Tris-HCl, $1 \mathrm{mM}$ EDTA, 2 $\mathrm{M} \mathrm{NaCl}, \mathrm{pH} 7.5)$ is self-configured with DIW water.

The RNA and DNA used in the experiment are shown in Table S1 (5'-3'), among which crRNA was synthesized and purified by Takara Biotechnology (Beijing, China) Co., Ltd., Aptamer 1, Aptamer 2, Trigger sequence and Tm probe sequence were Synthesized and purified by Sangon Biotechnology (Shanghai, China) Co., Ltd. (HPLC purification).

The characterization of element-labeled probe synthesis uses Thermo-LTQ-MS, and MALDI-TOF-MS (AXIMA Performance), and MALDI-TOF characterization uses 3 -Hydroxypyridine acid (3-HPA) as the substrate. A FluoChem M imager (Cell Biosciences; Santa Clara, CA) was used for gel electrophoresis imaging with an excitation wavelength of 300 nM. The reaction instrument mainly uses a gradient PCR instrument (K960, Shanghai HealForce Biological Co., Ltd.) and a constant temperature mixer (MSC-100, Hangzhou Allsheng Instrument Co., Ltd.). The stable isotope detection uses NexION 350 (PerkinElmer) ICPMS, and the operating conditions are shown in Table $\mathrm{S} 2$.

\section{EXPERIMENTAL SECTION}

Preparation of Tm Probe. $25 \mu \mathrm{L}$ of $\mathrm{TmCl}_{3}(20 \mathrm{mM})$ and $50 \mu \mathrm{L}$ of MMA-DOTA $(10 \mathrm{mM})$ are mixed first which both are dissolved and prepared with $\mathrm{CH}_{3} \mathrm{COONH}_{4}$ buffer $(500 \mathrm{mM}$, pH
$=5.5$ ). The mixed solution is placed at $37^{\circ} \mathrm{C}$ with slight shaking for $1 \mathrm{~h}$ to allow $\mathrm{Tm}^{3+}$ to fully chelate with MMA-DOTA. And then, the reaction mixture is mixed with the probe sequence (100 $\mu \mathrm{M}, \mathrm{CH}_{3} \mathrm{COONH}_{4}$ buffer dissolved) to react for $2 \mathrm{~h}$ at $37^{\circ} \mathrm{C}$ with slight shaking, where the MMA-DOTA is 25-fold excess to ensure complete capture of the probe sequence. The Tm probe initial solution is filtered and purified 5 times with an ultrafiltration tube (3k, Amicon Ultra-0.5 NMWL). Finally, the purified Tm probe was dissolved in HEPES buffer.

Procedure of the ICP-MS Detection. Take $10 \mu \mathrm{L}$ of $10 \times$ TOLO buffer in a $200 \mu \mathrm{L}$ centrifuge tube, add $20 \mu \mathrm{L}$ of the sample, 20 $\mu \mathrm{L}$ of the Trigger Locking hybrid DNA (diluted to $125 \mathrm{nM}$ with HEPES buffer), $8 \mu \mathrm{l}$ Cas12a/crRNA complex (Cas12a: crRNA $=1: 2), 5 \mu \mathrm{L}$ Tm probe, the final system is made up to $100 \mu \mathrm{L}$ with DEPC treated water. The mixed system was reacted at $25{ }^{\circ} \mathrm{C}$ temperature in a gradient PCR instrument for $90 \mathrm{~min}$, and then heated at $90{ }^{\circ} \mathrm{C}$ for $10 \mathrm{~min}$ to inactivate the enzyme.

Add an equal amount of $2 \times B \& W$ buffer to the system after the reaction is completed, mix, and add SA-MBs $\left(50 \mathrm{mg} \cdot \mathrm{mL}^{-1}\right)$. Incubate with shaking for $60 \mathrm{~min}$ at $25^{\circ} \mathrm{C}$. After the reaction, the SA-MBs were separated with a magnetic stand to remove intact Tm probe. The supernatant is acidified and diluted to $800 \mu \mathrm{L}$, and sent to ICP-MS for analysis.

\section{RESULTS AND DISCUSSION}

To confirm the chelation of $\mathrm{Tm}^{3+}$ and MMA-DOTA, we used ESI-MS to characterize the MMA-DOTA before and after the reaction (Fig. S1). After the reaction is complete, a peak with $\mathrm{M} / \mathrm{Z}$ of 711 is generated. It is proved that $\mathrm{Tm}^{3+}$ and MMA-DOTA can be successfully chelated under equal molar ratio. At the same time, whether the probe sequence is successfully connected to MMA-DOTA is characterized by MALDI-TOF-MS. Fig. S2 shows that peaks corresponding to their molecular weights appear in the reactant ssDNA (single strand DNA), ssDNA-DOTA and ssDNA -DOTA-Tm. And the peak of the reactant probe sequence almost disappeared in the latter two curves, indicating that under the condition of 25 -fold excess of MMA-DOTA, the probe sequence was almost completely captured in the probe. The two characterization proves that the Tm sequence was successfully synthesized. 


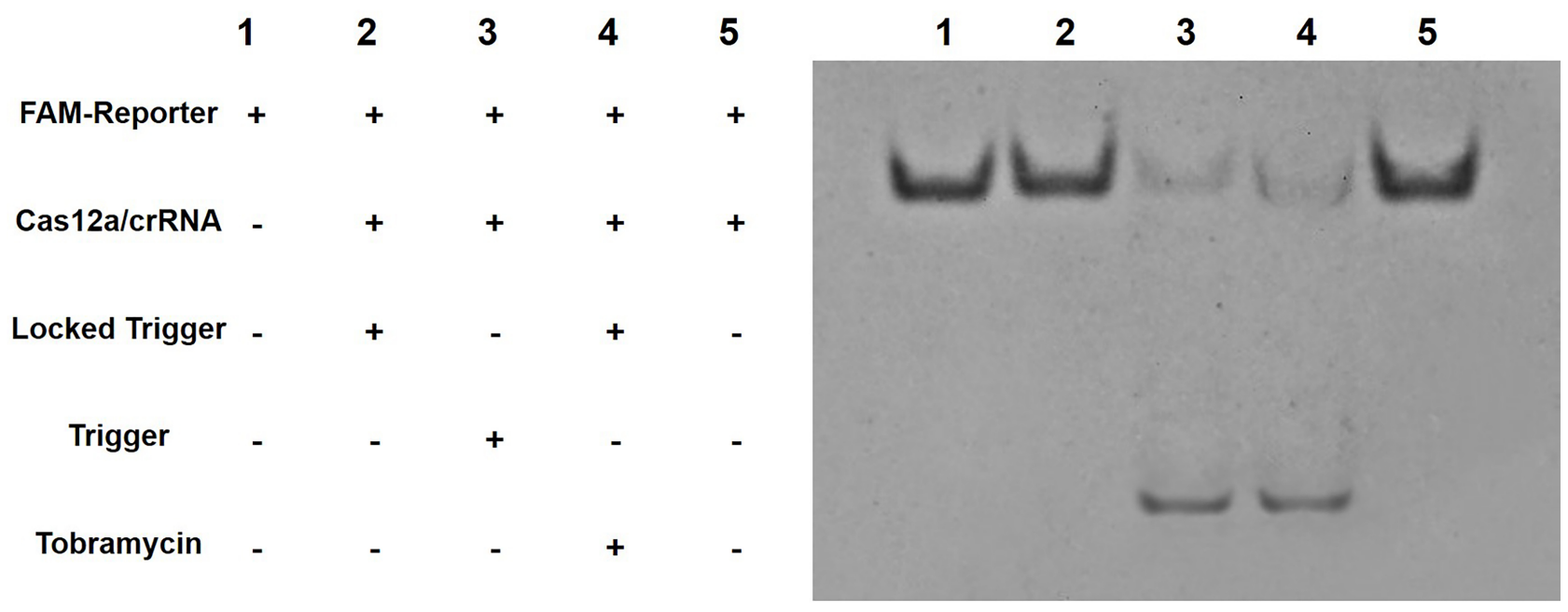

Fig. 3 PAGE analysis of the feasibility of aptamer-regulated CRISPR/Cas12a sensor for TOB detection.

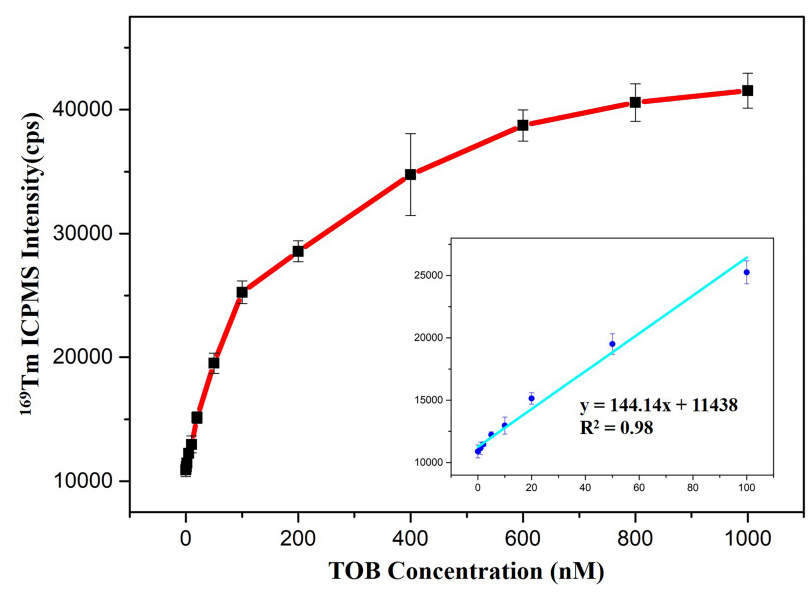

Fig. 4 The relationship between the ${ }^{169} \mathrm{Tm}$ ICP-MS signal and the tobramycin concentration; the inset is the linear curve between the tobramycin concentration and the ${ }^{169} \mathrm{Tm}$ ICP-MS signal.

The aptamer-regulated Cas12a-crRNA sensor was verified by polyacrylamide gel electrophoresis (PAGE), we use the fluorescent reporter probe (FAM-Reporter) used in the traditional CRISPR/Cas12a system as the cutting substrate ${ }^{23}$, and directly image the fluorescent reporter probe without adding nucleic acid stains. As shown in Fig. 3, when Trigger exists (lane 3), Cas12a/crRNA can stimulate the cleavage activity of FAM-reporter (lane 1), but there is no Trigger (lane 5) and locked Trigger (lane 2) cannot. After adding the target TOB, the locked Trigger can also be released (lane 4) to stimulate the cleavage activity of Cas12a/crRNA. This fully confirms the feasibility of the biosensor we designed for TOB detection.

Experiment conditions were optimized (Figs S3-S6). Afterward, the optimum concentrations of CRISPR/Cas12a, Tm probe, SA-MBs and the reaction time were employed for further experiments. Under the optimum conditions, the relationship of
Table 2. Comparison of the main analytical performances of the biosensing methods for the detection of TOB reported in the literature

\begin{tabular}{llll}
\hline Detection methods & Detection limit & Linear range & Ref. \\
\hline Fluorescent aptasensor & $21.86 \mathrm{nM}$ & $80-2000 \mathrm{nM}$ & 7 \\
LSPR aptasensor & $3.4 \mu \mathrm{M}$ & $0.5-80 \mu \mathrm{M}$ & 40 \\
$\begin{array}{l}\text { AuNPs aptasensor } \\
\begin{array}{l}\text { Photoelectrochemical } \\
\text { aptasensor }\end{array}\end{array}$ & $37.9 \mathrm{nM}$ & $100-1400 \mathrm{nM}$ & 41 \\
$\begin{array}{l}\text { Colorimetric } \\
\text { aptasensor }\end{array}$ & $4.28 \mathrm{nM}$ & $5-50 \mathrm{nM}$ & 42 \\
This work & $23.3 \mathrm{nM}$ & $40-200 \mathrm{nM}$ & 43 \\
\hline
\end{tabular}

${ }^{169} \mathrm{Tm}$ intensity and the concentration of TOB was investigated. As shown in Fig. 4, the linear range of the TOB concentration from 2.8 to $100 \mathrm{nM}$ was acquired with ${ }^{169} \mathrm{Tm}$ intensity. The linear equation between ${ }^{169} \mathrm{Tm}$ ICP-MS (Y, cps) and TOB concentration $(X, n M)$ was $Y=144.14 X+11438$, with the correlation coefficient $\mathrm{R}^{2}=0.98$. The detection limit is $0.83 \mathrm{nM}$ $(3 \sigma)$. The TOB biosensing method proposed in this study was compared with some recently reported biosensing methods for TOB detection. As shown in Table 2, the CRISPR/Cas12a TOB detection platform based on element probes proposed by this research has excellent sensitivity and analytical performance.

Since antibiotics are frequently used concomitantly, there are often multiple antibiotics with similar concentrations in detection, in practice, which is one of the difficulties in detection. In order to confirm the specificity of this experiment for TOB and to ensure that this biosensing strategy has sufficient anti-interference performance in actual detection, a series of antibiotics (Streptomycin, Gentamycin, Ampicillin, Tetracycline and Kanamycin) were selected as interfering substances in selectivity experiment (Fig. 5). The concentration of interfering antibiotics $(10 \mu \mathrm{M})$ is 100 times higher than TOB $(100 \mathrm{nM})$, and the mixture contains all of the above antibiotic ingredients. It can be seen that the specificity of the aptamer can overcome the interference of different antibiotics. 


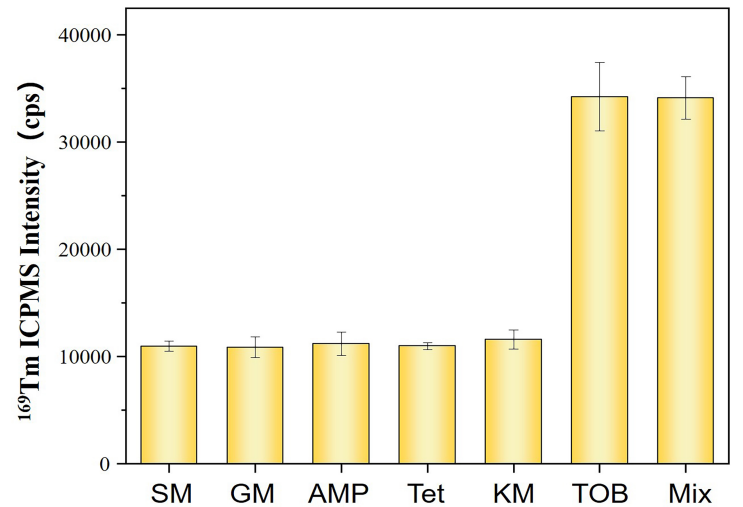

Fig. 5 Selectivity experiment of TOB detection, SM (Streptomycin), GM (Gentamycin), AMP (Ampicillin), Tet (Tetracycline), Kana (Kanamycin), TOB (Tobramycin), Mix (Mixture of all the above antibiotics).

For evaluating its reliability of the elemental probe-based CRISPR/Cas12a TOB biosensing, the real water sample verification is required. Five water samples were collected and spiked with TOB standard solutions of 10, 50, $100 \mathrm{nM}$ respectively. Water samples 1-5 were collected from 1. Tap water, 2. Pool water of Sichuan University (Sichuan, China), 3. Changbai Mountain Moya Spring (Jilin, China), 4. Emei Mountain Spring (Sichuan, China), 5. Jinjiang river (Chengdu, China). Table S3 shows that the recovery rate is between $95 \%$ and $103 \%$, and the RSD is between $1.7 \%$ and $4.3 \%$, which demonstrated the biosensing method we proposed to detect TOB has practical application potential.

\section{CONCLUSIONS}

In this work, a CRISPR/Cas12a TOB biosensor detection method based on element labeling was constructed, and a highly sensitive quantitative analysis of TOB was carried out. As the concentration of TOB increases, a correspondingly higher ICP-MS signal intensity can be obtained. This biosensing method for the detection of TOB has fabulous repeatability, the reaction time is as low as $90 \mathrm{~min}$, the detection limit can reach $0.83 \mathrm{nM}$, and the linear range of $2.8-100 \mathrm{nM}$ is satisfactory. In addition, this detection platform has significant specificity and potential practical application value for the detection of TOB. This novel biosensing method is expected to be applicable to the detection of more antibiotics and other markers with aptamers.

\section{ASSOCIATED CONTENT}

Supporting Information. The Supporting Information (Figs. S1-6, Tables S1-3) is available at www.at-spectrosc.com/as/home

\section{AUTHOR INFORMATION}

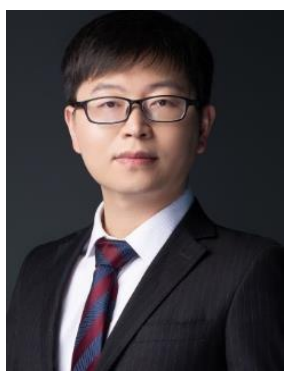

Rui Liu is a professor of analytical chemistry in the College of Chemistry of Sichuan University. He received his B.S. and Ph.D. degree from Sichuan University. During the graduate study, he received a scholarship from China Scholarship Council to work as visiting scholar in National Research Council Canada. After completing his Ph.D., he worked as a postdoctoral researcher in the Department of Chemistry in Tsinghua University. He joined the faculty of the College of Chemistry in Sichuan University in 2016. He has been working as member of editorial board for Atomic Spectroscopy. His current research interests are inductively coupled plasma mass spectrometry-based immunoassay and its applications in clinical diagnosis. He has published over sixty papers on scientific journals including Accounts of Chemical Research, Analytical Chemistry, and Atomic Spectroscopy.

\section{Corresponding Author}

* R. Liu

Email address: liur@scu.edu.cn

Notes

The authors declare no competing financial interest.

\section{ACKNOWLEDGMENTS}

The National Natural Science Foundation of China (22074096 and 22074098) is gratefully acknowledged. This work is also supported by the Talents Program of Sichuan Province (903) and the Fundamental Research Funds for the Central Universities. Dr. Xiaobo Xie from Analytical \& Testing Center, Sichuan University, and Dr. Chunxia Wang from College of Chemistry, Sichuan University, are thanked for the helpful discussion/ technical assistance.

\section{REFERENCES}

1. G. Andrzej and T. Magdalena, Asia-Pac. J. Ophthalmo., 2018, 7, 72-75. https://doi.org/10.22608/APO.2017343

2. W. Y. Mo, Z. T. Chen, H. M. Leung, and A. O. W. Leung, Environ. Sci. Pollut. R., 2017, 24, 8978-8989. https://doi.org/10.1007/s11356-015-5607-z

3. V. P. Hanko, J. S. Rohrer, H. H. Liu, C. M. Zheng, S. R. Zhang, X. J. Liu, and X. P. Tang, J. Pharmaceut. Biomed., 2008, 47, 828-833. https://doi.org/10.1016/j.jpba.2008.04.002

4. T. S. Medeiros, E. C. Pinto, L. M. Cabral, and V. P. Sousa, Microchem. J., 2021, 160, 105658. https://doi.org/10.1016/j.microc.2020.105658

5. X. Jin, L. Chen, Y. T. Zhang, X. L. Wang, and N. D. Zhou, Analyst, 2021, 146, 3608-3616. https://doi.org/10.1039/D1AN00403D 
6. A. Moga, M. Vergara-Barberán, M. J. Lerma-García, and E. J. Carrasco-Correa, J. M. Herrero-Martínez, and E. F. Simó-Alfonso, Compr. Rev. Food Sci. F., 2021, 20, 1681-1716. https://doi.org/ 10.1111/1541-4337.12702

7. Z. Khajavian, M. Esmaelpourfarkhani, M. Ramezani, M. Alibolandi, K. Abnous, and S. M. Taghdisi. Microchem. J., 2021, 160, 105657. https://doi.org/10.1016/j.microc.2020.105657

8. M. G. Pikkemaat, Anal. Bioanal. Chem., 2009, 395, 893-905. https://doi.org/10.1007/s00216-009-2841-6

9. M. X. Liu, Y. X. Sang, J. Zhang, J. Li, W. L. Yu, F. Y. Zhang, and X. H. Wang, B. Environ. Contam. Tox., 2021, 107, 215-220. https://doi.org/10.1007/s00128-020-02885-y

10. Y. X. Luan, N. Wang, C. Li, X. J. Guo, and A.X. Lu, Antibiotics- Basel, 2020 9, 787. https://doi.org/10.3390/antibiotics9110787

11. C. C. Wang, H. Li, N. Wang, H. D. Li, L. P. Fang, Z. Dong, H. X. Du, S. Guan, Q. Zhu, Z. L. Chen, and G. S. Yang, Anal. Methods, 2017, 9, 634-642. https://doi.org/10.1039/C6AY02921C

12. C. Pfeifer, G. Fassauer, H. Gerecke, T. Jira, Y. Remane, R. Frontini, J. Byrne, and R. Reinhardt, J. Chromatogr. B., 2015, 990, 7-14. https://doi.org/10.1016/j.jchromb.2015.02.043

13. L. M. Chiesa, M. Nobile, S. Panseri, and F. Arioli, Food Chem., 2017, 235, 111-118. https://doi.org/10.1016/j.foodchem.2017.04.184

14. M. Q. He, K. Wang, W. J. Wang, Y. L. Yu, and J. H. Wang, Anal. Chem., 2017, 89, 9292-9298. https://doi.org/10.1021/acs.analchem.7b02073

15. Y. Y. Wang, R. H. Zhou, W. X. Liu, C. Liu, and P. Wu, Chinese Chem. Lett., 2020, 31, 2950-2954. https://doi.org/10.1016/j.cclet.2020.01.023

16. Q. L Wen, J. Peng, A. Y. Liu, J. Wang, Y. L.Hu, J. Ling, and Q. E. Cao, Luminescence, 2020, 35, 702-708. https://doi.org/10.1002/bio.3775

17. C. Q. Wang, Z. Q. Deng, H. Zhang, R. Liu, and Y. Lv, Chinese Chem. Lett., 2022, 33, 1267-1270. https://doi.org/10.1016/j.cclet.2021.07.049

18. H. Kumar, K. Bhardwaj, T. Kaur, E. Nepovimova, K. Kuca, V. Kumar, S. K. Bhatia, D. S. Dhanjal, C. Chopra, R. Singh, S. Guleria, T. C. Bhalla, R. Verma, and D. Kumar, Foods, 2020, 9, 1504. https://doi.org/10.3390/foods9101504

19. F. Hille, H. Richter, S. P. Wong, M. Bratovic, S. Ressel, and E. Charpentier, Cell, 2018, 172, 1239-1259. https://doi.org/10.1016/j.cell.2017.11.032

20. J. Russel, R. Pinilla-Redondo, D. Mayo-Munoz, S. A. Shah, and S. J. Sorensen, CRISPR J., 2020, 3, 462-469. https://doi.org/10.1089/crispr.2020.0059

21. Y. L. Jiang, P. Yang, L. J. Du, L. Y. Xia, J. B. Chen, and X. D. Hou, Luminescence, 2021, 36, 1483-1490. https://doi.org/10.1002/bio.4087

22. M. McNutt, Breakthrough to genome editing. Science, 2015, 350, 1445. https://doi.org/10.1126/science.aae0479

23. Y. Xiong, J. J. Zhang, Z. L. Yang, Q. B. Mou, Y. Ma, Y. H. Xiong, and Y. Lu, J. Am. Chem. Soc., 2020, 142, 207-213. https://doi.org/10.1021/jacs.9b09211

24. M. M. Lv, J. W. Liu, R. Q. Yu, and J. H. Jiang, Chem. Sci., 2020, 11, 10361-10366. https://doi.org/10.1039/d0sc03698f

25. O. O. Abudayyeh, J. S. Gootenberg, S. Konermann, J. Joung, I. M. Slaymaker, D. B. Cox, S. Shmakov, K. S. Makarova, E. Semenova, L. Minakhin,
K. Severinov, A. Regev, E. S. Lander, E. V. Koonin, and F. Zhang, Science, 2016, 353, aaf5573. https://doi.org/10.1126/science.aaf5573

26. J. S. Gootenberg, O. O. Abudayyeh, M. J. Kellner, J. Joung, J. J. Collins, and F. Zhang, Science, 2018, 360, 439-444. https://doi.org/10.1126/science.aaq0179

27. P.-K. So, B.-C. Yang, W. Li, L. Wu, and B. Hu, Anal. Chem., 2019, 91 , 9430-9434. https://doi.org/10.1021/acs.analchem.9b02428

28. Z. Y. Li, R. Liu, and Y. Lv, Appl. Spectrosc. Rev., 2021. https://doi.org/10.1080/05704928.2021.1918703

29. R. Liu, S. X. Zhang, C. Wei, Z. Xing, S. C. Zhang, and X. R. Zhang, Accounts Chem. Res., 2016, 49, 775-783. https://doi.org/10.1021/acs.accounts.5b00509

30. Z. Y. Li, H. M. Li, D. Y. Deng, R. Liu, Y. Lv, Anal. Chem., 2020, 92, 4807-4813. https://doi.org/10.1021/acs.analchem.9b03995

31. Q. Li, Z. Q. Cai, Y. Fang, and Z. Wang, At. Spectrosc., 2021, 42, 238-246. https://doi.org/10.46770/AS.2021.101

32. Z. A. Hu, G. W. Sun, W. C. Jiang, F. J. Xu, Y. Q. Zhang, M. C. Xia, X. Y. Pan, Z. Xing, S. C. Zhang, and X. R. Zhang, Anal. Chem., 2019, 91, 5980-5986. https://doi.org/10.1021/acs.analchem.9b00405

33. M. Y. Pan, Y. Zang, X. R. Zhou, Y. L. Lu, J. P. Xiong, H. M. Li, and L. X. Feng, Atom. Spectrosc., 2021, 42, 262-270. https://doi.org/10.46770/AS.2021.104

34. N. S. Medvedev, A. V. Volzhenin, and A. I. Saprykin, At. Spectrosc., 2020, 42, 71-78. https://doi.org/10.46770/AS.2020.189

35. X. Chen, H. J. Song, Z. Y. Li, R. Liu, and Y. Lv, Anal. Chem., 2021, 93, 13719-13726. https://doi.org/10.1021/acs.analchem.1c03445

36. R. Nutiu and Y. F. Li, J. Am. Chem. Soc. 2003, 125, 4771-4778. https://doi.org/10.1021/ja028962o

37. X. Y. Fu, Y. Y. Shi, F. Q. Peng, M. Zhou, Y. Yin, Y. Tan, M. Chen, X. Yin, G. L. Ke, and X. B. Zhang, Anal. Chem., 2021, 93, 4967-4974. https://doi.org/10.1021/acs.analchem.1c00027

38. C. Zhang, F. B. Wu, Y. Y. Zhang, X. Wang, and X. R. Zhang, J. Anal. At. Spectrom., 2001, 16, 1393-1396. https://doi.org/10.1039/B106387C

39. X. Jin, L. M. Yang, X. W. Yan, and Q. Q. Wang, ACS Chem. Neurosci., 2021, 12, 1093-1099. https://doi.org/10.1021/acschemneuro.0c00816

40. G. Cappi, F. M. Spiga, Y. Moncada, A. Ferretti, M. Beyeler, M. Bianchessi, L. Decosterd, T. Buclin, and C. Guiducci, Anal. Chem., 2015, 87, 5278-5285. https://doi.org/10.1021/acs.analchem.5b00389

41. X. Y. Han, Y. H. Zhang, J. J. Nie, S. Y. Zhao, Y. P. Tian, and N. D. Zhou, Mikrochim Acta, 2017, 185, 4(2018). https://doi.org/10.1007/s00604-017-2568-6

42. X. Liu, Y. N. Jiang, J. Luo, X. Y. Guo, Y. Ying, Y. Wen, H. F. Yang, and Y. P. Wu, Food Chem., 2021, 344, 128716. https://doi.org/10.1016/j.foodchem.2020.128716

43. Q. Ma, Y. X. Wang, J. Jia, and Y. H. Xiang, Food Chem., 2018, 249, 98-103. https://doi.org/10.1016/j.foodchem.2018.01.022 